Pacific Journal of Mathematics

DIRECT LIMITS OF FINITE SPACES OF ORDERINGS 


\title{
DIRECT LIMITS OF FINITE SPACES OF ORDERINGS
}

\section{Mieczyslaw Kula, Murray A. Marshall and AndrzeJ SladeK}

\begin{abstract}
Spaces of orderings which are direct limits of finite spaces of orderings arise in a natural way. Every space of orderings is canonically a quotient space of such a space. In this paper we examine the internal structure of such spaces. In particular, we examine how the classification theory for finite spaces of orderings carries over to such spaces. We also establish a relationship between spaces of orderings which are direct limits of finite spaces and certain corresponding types of ultrasums of spaces of orderings. This has application to the problem of representing a space of orderings as the space of orderings of a Pythagorean field.
\end{abstract}

Generally, we use the terminology and notation of $[6,7,8]$. Throughout the paper, $X$ denotes a space of orderings and $G(X)$ denotes the underlying group of $X$. Thus $G(X)$ has exponent 2, and $X$ is a closed subset of the character group $\chi(G(X))$ satisfying the usual axioms. If $f$ is a (quadratic) form over $G(X)$, then $\operatorname{dim}(f)$ denotes the dimension of $f, \operatorname{dim}_{\mathrm{an}}(f)$ denotes the dimension of the anisotropic part of $f$, and $\sigma(f)$ denotes the signature of $f$ at $\sigma \in X$. We indicate that two forms $f, g$ are isometric (resp. Witt equivalent) by writing $f \cong g$ or $f \cong_{X} g$ (resp. $f \sim g$ or $f \sim_{X} g$ ). The value set of a form $f$ is denoted by $D(f)$ or $D_{X}(f)$. This consists of all $x \in G(X)$ satisfying $f \cong\left\langle x, x_{2}, \ldots, x_{n}\right\rangle$ for some $x_{2}, \ldots, x_{n} \in G(X)$. The sum and product of forms $f, g$ is denoted by $f \oplus g$ and $f \otimes g$, and if $n$ is a positive integer, then $n \times f=f \oplus \cdots \oplus f$ ( $n$ times). If $Y$ is also a space of orderings, then a morphism from $X$ to $Y$ is a map $\varphi^{*}: X \rightarrow Y$ induced by a group homomorphism $\varphi: G(Y) \rightarrow G(X)$ in the sense that $\varphi^{*}(\sigma)=\sigma \circ \varphi$ for all $\sigma \in X$.

The Witt ring of $X$ is denoted by $W(X)$. If $\sigma \in X$, the ring homomorphism from $W(X)$ into $\mathbf{Z}$ induced by $\sigma$ is also denoted by $\sigma$. By $[\mathbf{8}$, Lem. 7.1], $G(X)$ is naturally identified with the group of units of $W(X)$. Also, it is well known (and easily verified, e.g., using [8, Lem. 4.1]) that $X$ is identified with the set of all ring homomorphisms from $W(X)$ into $\mathbf{Z}$. Also, if $\varphi^{*}: X \rightarrow Y$ is a morphism of spaces of orderings then the associated group homomorphism $\varphi: G(Y) \rightarrow G(X)$ induces a ring homomorphism (also denoted by $\varphi$ ) from $W(Y)$ into $W(X)$. Every ring homomorphism from $W(Y)$ into $W(X)$ is of this form for some unique morphism $\varphi^{*}: X \rightarrow Y$. In this way the category of Witt rings of spaces of 
orderings and the category of spaces of orderings are equivalent. For a ring-theoretic treatment of Witt rings of spaces of orderings, we refer the reader to $[3, \mathbf{1 0}]$.

1. Direct limits of finite spaces. As in [7] we will denote the direct sum of a finite collection of spaces of orderings $\left\{X_{1}, \ldots, X_{n}\right\}$ by $X_{1}$ $\oplus \cdots \oplus X_{n}$. The direct sum of an arbitrary collection of spaces of orderings $\left\{X_{l}: i \in I\right\}$ also exists and will be denoted by $\sum_{l \in I} X_{i}$. This is the space of orderings $X$ defined as follows: $G(X)$ is $\prod_{l \in I} G\left(X_{i}\right)$, the direct product in the category of groups. The projection $G(X) \rightarrow G\left(X_{l}\right)$ identifies $X_{i}$ with a closed subset of the character group $\chi(G(X))$ and $X_{t} \cap X_{J}=\varnothing$ if $i \neq j . X$ is defined to be the closure of $\dot{\cup}_{l \in I} X_{l}$ in $\chi(G(X))$. Note if $I$ is finite then $\dot{\cup}_{l \in I} X_{l}$ is already closed. If $f$ is a form over $G(X)$, we denote by $f_{l}$ the associated form over $G\left(X_{l}\right)$. Since forms over $G(X)$ define continuous functions on $X$, it is clear that $f \sim g \Leftrightarrow f_{t} \sim g_{i}$ for all $i \in I$. The proof that $X$ is a space of orderings and the direct sum of the collection $\left\{X_{l}: i \in I\right\}$ in the category of spaces of orderings is straightforward and will be omitted. $W(X)$ is identified with a subring of the direct product ring $\Pi_{l \in I} W\left(X_{i}\right)$. Observe that $W(X)$ consists of all elements $\left(f_{l}\right)_{i \in I}$ in $\prod_{l \in I} W\left(X_{l}\right)$ satisfying:

(i) $\operatorname{dim}\left(f_{l}\right) \equiv \operatorname{dim}\left(f_{j}\right)(\bmod 2)$ for all $i, j \in I$, and

(ii) $\sup \left\{\operatorname{dim}_{\text {an }}\left(f_{l}\right): i \in I\right\}<\infty$.

If $I$ is finite then (ii) is vacuous.

We consider direct limits (of directed systems) of finite spaces of orderings. It turns out that these always exist in the category of spaces of orderings. Let $\left(X_{l}: i \in I\right)$ be a directed system of finite spaces of orderings. Thus $I$ is an ordered set and morphisms $\varphi_{l j}^{*}: X_{l} \rightarrow X_{j}$ are specified whenever $i \leq j$. These are assumed to satisfy $\varphi_{j k}^{*} \circ \varphi_{l j}^{*}=\varphi_{l k}^{*}$ whenever $i \leq j \leq k$. It is also assumed that $I$ is directed, i.e., that for all $i, j \in I$, there exists $k \in I$ such that $i, j \leq k$. To define $X:=\lim _{\rightarrow \in I} X_{l}$ we first use the associated group homomorphism $\varphi_{l}: G\left(X_{j}\right) \rightarrow G\left(X_{l}\right)$ to form $G(X):=\lim _{\iota \in I} G\left(X_{l}\right)$, the inverse limit in the category of groups. The group homomorphism $\varphi_{l}: G(X) \rightarrow G\left(X_{i}\right)$ induces a group homomorphism $\varphi_{1}^{*}: \chi\left(G\left(X_{l}\right)\right) \rightarrow \chi(G(X))$, and $X$ is defined to be the closure of $\cup_{t \in I} \varphi_{i}^{*}\left(X_{i}\right)$ in $\chi(G(X))$.

THEOREM 1.1. For any directed system $\left(X_{i}: i \in I\right)$ of finite spaces of orderings, $X$ as defined above is a space of orderings, and $X$ is the direct limit of the directed system $\left(X_{i}: i \in I\right)$ in the category of spaces of orderings.

Proof. Let $Y=\sum_{l \in I} X_{l}$. Then $G(X) \subseteq G(Y)$ and $X$ is the image of $Y$ under the restriction map $\chi(G(Y)) \rightarrow \chi(G(X))$. We prove $X$ is a space of 
orderings using [7, Lem. 3.3]. First note, since each $G\left(X_{i}\right)$ is finite, $G(Y)$, with the product topology, is a compact totally disconnected topological group. For $i \leq j$ define $V_{i j}=\left\{x \in G(Y): \varphi_{i j}\left(x_{j}\right)=x_{i}\right\}$. This is closed in $G(Y)$ and $G(X)=\cap\left\{V_{l j}: i, j \in I, i \leq j\right\}$. Also, if $f$ is a form over $G(Y)$, then the value set $D_{Y}(f)=\prod_{i \in I} D_{X_{1}}\left(f_{i}\right)$ is closed in $G(Y)$. Now suppose $f, g$ are forms over $G(X)$ and $D_{Y}(f) \cap D_{Y}(g) \neq \varnothing$. Thus the sets $D_{Y}(f) \cap D_{Y}(g) \cap V_{i j}$ are closed in $G(Y)$ and using the fact that $I$ is directed and the fact that the maps $\varphi_{i,}$ preserve value sets, one verifies that the intersection of finitely many such sets is not empty. Thus, by compactness, $D_{Y}(f) \cap D_{Y}(g) \cap G(X) \neq \varnothing$. It follows from [7, Lem. 3.3] that $X$ is a space of orderings. The proof that $X$ is the direct limit of the directed system $\left(X_{i}: i \in I\right)$ in the category of spaces of orderings is straightforward and will be omitted.

Corollary 1.2. Suppose $X=\lim _{\rightarrow \in I} X_{i}$ where each $X_{t}$ is finite. Then for any form $f$ over $G(X)$,

(i) $f \sim 0 \Leftrightarrow f_{i} \sim 0$ for all $i \in I$,

(ii) $f$ is $X$-isotropic $\Leftrightarrow f_{l}$ is $X_{i}$-isotropic for all $i \in I$,

(iii) $D_{X}(f)=\lim _{i \in I} D_{X_{i}}\left(f_{l}\right)$.

Proof. Let $Y=\sum_{i \in I} X_{l}$. The first assertion is clear. By [7, Rem. 3.4] and the proof of Theorem 1.1, $f$ is $X$-isotropic $\Leftrightarrow f$ is $Y$-isotropic. On the other hand it is clear that $f$ is $Y$-isotropic $\Leftrightarrow f_{i}$ is $X_{i}$-isotropic for all $i \in I$. Also, by the proof of [7, Lem. 3.3], $D_{X}(f)=D_{Y}(f) \cap G(X)$. Since $D_{Y}(f)=\prod_{i \in I} D_{X_{t}}\left(f_{l}\right)$, and $G(X)=\lim _{\imath \in I} G\left(X_{i}\right)$, this implies $D_{X}(f)=$ $\lim _{\imath \in I} D_{X_{i}}\left(f_{i}\right)$.

THEOREM 1.3. Suppose $X=\lim _{\rightarrow \in I} X_{\imath}$ where each $X_{i}$ is finite. Then $W(X)$ is the subring of the ring-theoretic inverse limit $\lim _{i \in I} W\left(X_{l}\right)$ consisting of all $\left(f_{l}\right)_{i \in I}$ satisfying $\sup \left\{\operatorname{dim}_{\text {an }}\left(f_{i}\right): i \in I\right\}<\infty$.

Proof. We use the notation of Theorem 1.1. It is clear that $W(X)$ $\subseteq \lim _{\leftarrow} W\left(X_{i}\right)$ and every element $\left(f_{l}\right)_{t \in I}$ of $W(X)$ satisfies $\sup \left\{\operatorname{dim}_{\mathrm{an}}\left(f_{i}\right)\right.$ : $i \in \overleftarrow{I\}}<\infty$. Since $I$ is directed and $\varphi_{i j}\left(f_{j}\right) \sim f_{l} \Rightarrow \operatorname{dim}\left(f_{i}\right) \equiv \operatorname{dim}\left(f_{j}\right)$ $(\bmod 2)$, we know that every element of $\lim _{\iota \in I} W\left(X_{l}\right)$ satisfying this latter condition is represented by a form over $G(Y)$ so we are reduced to proving the following Claim: Suppose $f$ is a form over $G(Y)$ satisfying $\varphi_{i j}\left(f_{j}\right) \cong f_{l}$ for all $i, j \in I$ such that $i \leq j$. Then there exists a form $g$ over $G(X)$ satisfying $f \cong g$. For by hypothesis, any intersection of finitely many of the closed sets $D_{Y}(f) \cap V_{i j}$ is not empty. Thus, by compactness 
of $G(Y), \quad D_{Y}(f) \cap G(X) \neq \varnothing$, say $x_{1} \in D_{Y}(f) \cap G(X)$. Thus $f \cong$ $\left\langle x_{1}\right\rangle \oplus g$ where $g$ is a form over $G(Y)$ of dimension $n-1$, where $n=\operatorname{dim}(f)$. Since $g$ satisfies the same hypothesis as $f$, by induction on $n$, there exist $x_{2}, \ldots, x_{n} \in G(X)$ such that $g \cong\left\langle x_{2}, \ldots, x_{n}\right\rangle$, so $f \cong$ $\left\langle x_{1}, \ldots, x_{n}\right\rangle$.

A space of orderings which is expressible as a direct limit of finite spaces of orderings will be referred to as a DLO finite space for short. Note, if $X$ is a DLO finite space of orderings then, with the inverse limit topology, $G(X)$ is a compact totally disconnected topological group and $W(X)$ is a totally disconnected topological ring.

Remark 1.4. Suppose $X$ is any space of orderings. The subspace of $X$ generated by a finite set $\left\{\sigma_{1}, \ldots, \sigma_{n}\right\} \subseteq X$ consists of all $\sigma \in X$ expressible as $\sigma=\sigma_{1}^{e_{1}} \cdots \sigma_{n}^{e_{n}}$ where $e_{1}, \ldots, e_{n} \in\{0,1\}$. In particular, this subspace is finite. Thus, the finite subspaces of $X$ form a directed system when ordered by inclusion. Suppose we denote the direct limit of this system by $\hat{X}$. Let $\varphi^{*}: \hat{X} \rightarrow X$ be the natural morphism which exists by the universal property of $\hat{X}$. This is surjective and hence identifies $X$ with a quotient space of $\hat{X}$. The corresponding inclusions $\varphi: G(X) \rightarrow G(\hat{X})$ (resp. $\varphi$ : $W(X) \rightarrow W(\hat{X})$ ) identify $G(X)$ (resp. $W(X)$ ) with a dense subgroup of $G(\hat{X})$ (resp. with a dense subring of $W(\hat{X})$ ). Since the results in [2] are a bit confusing on this point, we emphasize that $\varphi$ is not surjective except in the trivial case where $X$ is finite. Note also, by [9, Th. 1.4] and Cor. 1.2 (ii), if $f$ is a form over $G(X)$, then $\varphi(f)$ is $\hat{X}$-isotropic iff $f$ is $X$-isotropic.

Suppose $X$ is DLO finite, say $X=\lim _{i \in I} X_{l}$. Let $X^{c}:=\cup_{i \in I} \varphi_{l}^{*}\left(X_{l}\right)$ where $\varphi_{l}^{*}: X_{i} \rightarrow X$ is the induced morphism. Thus $X^{c}$ is dense in $X$. Note that the kernel of $\varphi_{i}: G(X) \rightarrow G\left(X_{i}\right)$ (resp. $\varphi_{i}: W(X) \rightarrow W\left(X_{i}\right)$ ) is the intersection of the kernels of $\sigma \circ \varphi_{l}: G(X) \rightarrow\{ \pm 1\}$ (resp. $\sigma \circ \varphi_{l}: W(X) \rightarrow$ Z), $\sigma \in X_{i}$. Thus the inverse limit topology on $G(X)$ (resp. $\left.W(X)\right)$ is the weakest topology such that the map $\sigma: G(X) \rightarrow\{ \pm 1\}($ resp. $\sigma: W(X) \rightarrow \mathbf{Z})$ is continuous for each $\sigma \in X^{c}$. The following theorem shows, in particular, that $X^{c}$ depends only on the topology on $G(X)$ (resp. $W(X)$ ), i.e., is independent of the particular presentation of $X$ as a direct limit of finite spaces.

Theorem 1.5. Suppose $X$ is DLO finite and $\sigma \in X$. Then the following are equivalent:

(i) $\sigma \in X^{c}$,

(ii) $\sigma: W(X) \rightarrow \mathbf{Z}$ is continuous,

(iii) $\sigma: G(X) \rightarrow\{ \pm 1\}$ is continuous. 
Proof. We use the notation of the proof of Theorem 1.1. The implications (i) $\Rightarrow$ (ii) and (ii) $\Rightarrow$ (iii) are trivial. To prove (iii) $\Rightarrow$ (i) suppose $\sigma$ : $G(X) \rightarrow\{ \pm 1\}$ is continuous. Since $G(X)=\lim _{\iota \in I} G\left(X_{i}\right)$, there exists $i \in I$ and a character $\tau$ on $G\left(X_{l}\right)$ such that $\sigma=\tau \circ \varphi_{i}$. For $j \geq i$ define the character $\sigma_{j}$ on $G\left(X_{j}\right)$ by $\sigma_{J}=\tau \circ \varphi_{i j}$. If $\sigma_{J} \in X_{J}$ for some $j \geq i$, we are done. Assume this is not the case. Then by [8, Lem. 4.1], there exist $a_{j}, b_{j} \in G\left(X_{j}\right)$ such that $b_{j} \in D_{X_{j}}\left\langle 1, a_{j}\right\rangle, \sigma_{j}\left(a_{j}\right)=1, \sigma_{j}\left(b_{j}\right)=-1$. For each $j \geq i$ let $W_{j}$ denote the set of $(a, b) \in G(Y) \times G(Y)$ such that $b_{j} \in D_{X_{j}}\left\langle 1, a_{j}\right\rangle, \sigma_{j}\left(a_{j}\right)=1$, but $\sigma_{j}\left(b_{j}\right)=-1$. This is closed in $G(Y) \times$ $G(Y)$ so for each $j \geq i$ and each $k \leq j$, the sets $W_{j} \cap\left(V_{j k} \times V_{j k}\right)$ are closed in $G(Y) \times G(Y)$. One checks that the intersection of finitely many of these sets is not empty, so by compactness of $G(Y) \times G(Y)$, there exist $a, b \in G(X)$ such that $b \in D_{X}\langle 1, a\rangle, \sigma(a)=1$, but $\sigma(b)=-1$. This contradicts $\sigma \in X$.

Corollary 1.6. Suppose $X, Y$ are DLO finite and $\varphi^{*}: X \rightarrow Y$ is a morphism. Then the following are equivalent:

(i) $\varphi^{*}\left(X^{c}\right) \subseteq Y^{c}$,

(ii) $\varphi: W(Y) \rightarrow W(X)$ is continuous,

(iii) $\varphi: G(Y) \rightarrow G(X)$ is continuous.

Proof. The topology on $W(X)$ is the weakest such that each $\sigma \in X^{c}$ defines a continuous function on $W(X)$, so (i) $\Rightarrow$ (ii). (ii) $\Rightarrow$ (iii) is trivial. Finally, (iii) $\Rightarrow$ (i) follows from Theorem 1.5.

Although a DLO finite space $X$ may have several presentations as a direct limit of finite spaces, there is a standard presentation. To obtain this we denote by $\mathscr{U}(X)$ the set of all finite subspaces of $X$ which lie in $X^{c}$.

Corollary 1.7. Suppose $X$ is DLO finite. Then $\mathcal{Q}(X)$ is a directed system of finite spaces of orderings and $X$ is the direct limit of this system.

Proof. Since a finite product of continuous characters on $G(X)$ is again a continuous character on $G(X)$, it follows using Theorem 1.5 and the argument in Remark 1.4 that the finite subspace of $X$ generated by $\sigma_{1}, \ldots, \sigma_{n} \in X^{c}$ lies in $X^{c}$. This proves that $\mathscr{Q}(X)$ is directed. The image of $G(X)$ in $\lim _{Y \in \mathcal{Q}(X)} G(Y)$ via the natural map is dense and since the map is continuous and $G(X)$ is compact, the image is closed. Thus the map is surjective. It is injective since $X^{c}$ is dense in $X$. It should be clear now that $X$ is the direct limit of the directed system $\mathcal{Q}(X)$. 
We are now in a position to characterize DLO finite spaces internally.

COROLlarY 1.8. Suppose $X$ is a space of orderings and $G(X)$ has the structure of a compact topological group such that $X^{c}:=\{\sigma \in X: \sigma$ is continuous on $G(X)\}$ is dense in $X$. Then $X$ is DLO finite and the topology on $G(X)$ is the induced inverse limit topology.

Proof. Immediate from the proof of Corollary 1.7.

The content of Corollary 1.8 may also be phrased as follows:

COROLlaRY 1.9. Let $X$ be a space of orderings. Then $X$ is DLO finite iff there exists a subset $B \subseteq X$ satisfying:

(i) $[B] \cap X$ is dense in $X$. (Here, $[B]$ denotes the linear span of $B$ in $\chi(G(X))$.)

(ii) The natural map $\varphi: G(X) \rightarrow\{ \pm 1\}^{B}$ is onto. (Note, $\varphi$, is already 1-1 by (i).)

Proof. Suppose $X$ has a subset $B$ satisfying (i) and (ii). Then the isomorphism $\varphi$ induces a topology on $G(X)$ giving it the structure of a compact totally disconnected topological group. Also, $X^{c}=[B] \cap X$ is dense in $X$, so $X$ is DLO finite by Corollary 1.8. Observe that $B$ is a maximal linearly independent subset of $X^{c}$. Conversely, if $X$ is DLO finite and $B$ is any maximal linearly independent subset in $X^{c}$, then $[B] \cap X=$ $X^{c}$ is dense in $X$ and $\varphi$ is continuous and has dense image in $\{ \pm 1\}^{B}$ so by compactness of $G(X), \varphi$ is onto.

2. Decomposition of DLO finite spaces. In this section we show how the structure theory of finite spaces of orderings given in [6] carries over to DLO finite spaces of orderings which satisfy certain finiteness conditions. Throughout, we work in the category consisting of DLO finite spaces together with morphisms which are continuous in the sense of Corollary 1.6.

If $\Delta$ is any compact totally disconnected topological group of exponent 2 , we denote by $\chi^{c}(\Delta)$ the group of continuous characters on $\Delta$. Note, for any such $\Delta, \Delta \cong\{ \pm 1\}^{\alpha}$, where $\alpha$ denotes the $\mathbf{Z} / 2 \mathbf{Z}$ dimension of $\chi^{c}(\Delta)$.

THEOREM 2.1. Suppose $X$ is the group extension of $Y$ by the group $\Delta$ in the sense of [7] where $Y$ is DLO finite and $\Delta$ is compact totally disconnected with exponent 2. Then $X$ has, in a natural way, the structure of a DLO finite space. 
Proof. By definition, $G(X)=G(Y) \times \Delta$ and $X=Y \times \chi(\Delta)$. Giving $G(X)$ the product topology, $X^{c}=Y^{c} \times \chi^{c}(\Delta)$, and $X$ is DLO finite using Corollary 1.8.

For any space of orderings $X$, the translation group $\operatorname{gr}(X)$ is defined as in [9]. For $X$ DLO finite, the group to consider is $\operatorname{gr}\left(X^{c}\right):=\{\gamma \in$ $\left.\chi^{c}(G(X)): \gamma X^{c}=X^{c}\right\}$. Using the density of $X^{c}$ in $X$ and the modification of [6, Lem. 4.2] mentioned in [9, Rem. 2.1], it follows that $\operatorname{gr}\left(X^{c}\right)=\operatorname{gr}(X)$ $\cap \chi^{c}(G(X))$. Note if $X$ is the group extension of $Y$ by $\Delta$ as in Theorem 2.1, then $\operatorname{gr}\left(X^{c}\right)=\operatorname{gr}\left(Y^{c}\right) \times \chi^{c}(\Delta)$ and $Y$ is the residue space of $X$ corresponding to $\chi^{c}(\Delta) \subseteq \operatorname{gr}\left(X^{c}\right)$. Conversely, we have the following:

THEOREM 2.2. Suppose $X$ is DLO finite and $H$ is any subgroup of $\operatorname{gr}\left(X^{c}\right)$. Then the residue space $Y$ of $X$ corresponding to $H$ is $D L O$ finite and $X$ is the group extension of $Y$ by a compact totally disconnected group $\Delta \cong \chi(H)$ as in Theorem 2.1. In particular, if $H=\operatorname{gr}\left(X^{c}\right)$, then $\operatorname{gr}\left(Y^{c}\right)=1$.

Proof. By definition $Y$ is the quotient space of $X$ obtained by restricting elements of $X$ to $G(Y):=\{a \in G(X): \gamma(a)=1$ for all $\gamma \in H\}$. This is closed in $G(X)$ since each $\gamma \in H$ is continuous. $Y$ is DLO finite by an easy application of Corollary 1.8. Also, since $G(Y)$ is a closed subgroup of $G(X)$, there exists a closed subgroup $\Delta \subseteq G(X)$ such that $G(X)=G(Y) \times \Delta$. The rest of the assertions are clear.

THeOREM 2.3. Suppose $X_{i}$ is DLO finite for all $i \in I$. Then $X=\sum_{l \in I} X_{l}$ is DLO finite and $X^{c}=\dot{U}_{l \in I} X_{i}^{c}$.

Proof. $G(X)=\prod_{i \in I} G\left(X_{i}\right)$ is compact totally disconnected with the product topology. Since $\dot{\cup}_{l \in I} X_{i}$ is dense in $X$ and $X_{l}^{c}$ is dense in $X_{i}$, $\dot{\cup}_{i \in I} X_{i}^{c}$ is dense in $X$. $\dot{\cup}_{i \in I} X_{i}^{c} \subseteq X^{c}$, so $X^{c}$ is dense in $X$. Thus $X$ is DLO finite by Corollary 1.8. Suppose $\sigma \in X^{c}$. By continuity, $\sigma$ factors through $\Pi_{\imath \in J} G\left(X_{l}\right)$ for some finite set $J \subseteq I$. Let $Y=\Sigma_{l \in J} X_{\imath}$ and $Z=\Sigma_{\imath \in K} X_{i}$ where $K=I \backslash J$. Then $X=Y \oplus Z$ so by choice of $J, \sigma \in Y$. Since $J$ is finite there exists $i \in J$ such that $\sigma \in X_{i}$. Using the continuity of $\sigma$ once more, this implies $\sigma \in X_{l}^{c}$.

For $X$ DLO finite, we define a relation $\sim_{c}$ on $X^{c}$ by declaring $\sigma \sim_{c} \tau$ to mean that either $\sigma=\tau$ or there exists a 4-element fan $V \subseteq X^{c}$ with $\sigma, \tau \in V$. Thus if $\mathscr{U}(X)$ is defined as in Corollary 1.7, then for $\sigma, \tau \in X^{c}$, $\sigma \sim_{c} \tau$ holds iff $\sigma, \tau$ lie in the same connected component of $Y$ for some $Y \in \mathscr{Q}(X)$. Thus, by results in $[6,9], \sim_{c}$ is an equivalence relation on $X^{c}$. 
The equivalence classes of $X^{c}$ with respect to $\sim_{c}$ are called the connected components of $X^{c}$. We say $X^{c}$ is connected if it has only one connected component.

THEOREM 2.4. Every DLO finite space $X$ decomposes uniquely as $X \cong \sum_{i \in I} X_{l}$ where $X_{l}^{c}$ is connected for each $i \in I$.

Proof. If $X=\sum_{i \in I} X_{i}$ is such a decomposition then, by Theorem 2.3, $X^{c}=\dot{\cup}_{t \in I} X_{i}^{c}$, so by [8, Cor. 7.5(i)], the sets $X_{i}^{c}, i \in I$, are the connected components of $X^{c}$ and $X_{t}$ is the subspace of $X$ obtained by closing $X_{i}^{c}$ topologically. This proves uniqueness.

To prove existence, let $\left\{C_{l}: i \in I\right\}$ be the connected components of $X^{c}$. For each $Y \in \mathcal{U}(X)$, let $I(Y)=\left\{i \in I: Y \cap C_{l} \neq \varnothing\right\}$. Thus $Y=$ $\sum_{i \in I(Y)} Y$, where $Y_{i}=Y \cap C_{i}$. This follows from the decomposition theory in $[6,9]$. For $i \in I$, let $\mathscr{Q}(X)_{i}=\left\{Y \in \mathcal{U}(X): Y \subseteq C_{i}\right\}$. Note $\mathscr{U}(X)_{l}$, ordered by inclusion, is a directed system of finite spaces of orderings. Denote by $X_{l}$ the direct limit of this system. Observe that the inclusions $Y \subseteq X$ for $Y \in \mathscr{Q}(X)_{i}$ together with the universal property of $X_{l}$ yields a morphism $\psi_{l}^{*}: X_{l} \rightarrow X$ for each $i \in I$ and hence a morphism $\psi^{*}: \Sigma_{l \in I} X_{i} \rightarrow$ $X$. On the other hand, for each $Y \in \mathcal{U}(X), Y=\Sigma_{l \in I(Y)} Y_{i}$ as above, so the inclusions $Y_{i} \subseteq X_{i}$ induce a morphism $Y \rightarrow \Sigma_{l \in I(Y)} X_{i} \rightarrow \Sigma_{i \in I} X_{l}$. By the universal property of $X$ this lifts to a morphism $\varphi^{*}: X \rightarrow \sum_{l \in I} X_{l}$. It is clear that $\psi^{*} \circ \varphi^{*}=1$ and $\varphi^{*} \circ \psi^{*}=1$ so $X \cong \Sigma_{i \in I} X_{l}$.

We denote the stability index of a space of orderings $X$ by $\operatorname{st}(X)$. Various characterizations of $\operatorname{st}(X)$ are given in $[\mathbf{8}, \mathbf{1 1}]$. Suppose $X$ is DLO finite and $\mathcal{U}(X)$ is defined as in Corollary 1.7. If $Y \in \mathcal{U}(X)$, then $\operatorname{st}(Y) \leq \operatorname{st}(X)$. On the other hand, if $p$ is an anisotropic Pfister form over $G(X)$ and if, for each $Y \in \mathcal{U}(X)$, there exists a form $q_{Y}$ over $G(X)$ such that $p \cong_{Y} 2 \times q_{Y}$, then, by Theorem 1.3, there exists a form $q$ over $G(X)$ such that $p \cong_{X} 2 \times q$. It follows from [8, Th. 6.2] that $\operatorname{st}(X)=\sup \{\operatorname{st}(Y)$ : $Y \in \mathcal{U}(X)\}$.

THEOREM 2.5. For $X$ DLO finite and $n \geq 0$, the following are equivalent:

(i) $\operatorname{st}(X) \leq n$,

(ii) $X \in \mathscr{P}_{n}$ where $\mathscr{P}_{0}$ denotes the class of singleton spaces and, inductively, $\mathscr{P}_{k+1}$ consists of all DLO finite spaces $Y$ expressible in the form $Y \cong\left(\sum_{l \in I} Y_{i}^{(1)}\right) \oplus\left(\Sigma_{j \in J}\left\{\sigma_{j}\right\}\right)$ where $Y_{i} \in \mathscr{P}_{k}$ for all $i \in I$. (Here, $Y_{l}^{(1)}$ denotes the group extension of $Y_{i}$ by $\{ \pm 1\}$ and $\left\{\sigma_{j}\right\}$ denotes a singleton space.) 
Proof. (ii) $\Rightarrow$ (i): If $Y$ is as in (ii) and $k \geq 0$, then $\operatorname{st}(Y) \leq k+1 \Leftrightarrow$ $\operatorname{st}\left(Y_{l}\right) \leq k$ for all $i \in I$. Thus, by induction, if $X \in \mathscr{P}_{n}$ then st $(X) \leq n$.

(i) $\Rightarrow$ (ii): Suppose $X$ is DLO finite, $\operatorname{st}(X) \leq n$. By Theorems 2.2, 2.4, and induction on $n$, it is enough to show that if $X^{c}$ is connected and $\left|X^{\prime}\right| \neq 1$, then $\operatorname{gr}\left(X^{c}\right) \neq 1$. Since $\operatorname{st}(Y) \leq \operatorname{st}(X) \leq n$ for all $Y \in \mathcal{Q}(X)$, it follows from the proof of $[9$, Th. 2.6] that there is a uniform bound on the length of chains

$$
Y_{\gamma_{0}} \subsetneq Y_{\gamma_{1}} \subsetneq \cdots \subsetneq Y_{\gamma_{l}}
$$

where $Y \in \mathcal{Q}(X), \gamma_{l} \neq 1, \gamma_{l} \in \chi^{c}(G(X))$, and $Y_{\gamma_{l}}$ is a non-fan for $i=$ $0, \ldots$, l. Suppose $(*)$ is a chain of this type of maximal length. We claim $\gamma_{l} \in \operatorname{gr}\left(X^{c}\right)$. For let $\sigma \in X^{c}$. Since $X^{c}$ is connected, there is a finite subspace $Z \subseteq X^{c}$ with $Y \subseteq Z, \sigma \in Z$, and with $\sigma$ and $Y_{\gamma_{1}}$ lying in the same connected component of $Z$. Since $Y$ is a subspace, $Y_{\gamma_{l}}=Z_{\gamma_{l}} \cap Y$. Thus $Z_{\gamma_{t}}$ is a non-fan for $i=0, \ldots, l$ and these sets are linearly ordered by inclusion by [11, Th. 1.3]. From this and (*) it follows that $Z_{\gamma_{1-1}} \subsetneq Z_{\gamma_{1}}$ for $i=1, \ldots, l$. By the maximal choice of $l$ and the structure theory in the finite case, $Z_{\gamma_{l}}$ is a connected component in $Z$. Thus $\sigma \in Z_{\gamma_{l}}$. In particular, this implies $\sigma \gamma_{l} \in X^{c}$. Since $\sigma \in X^{c}$ is arbitrary, this proves $\gamma_{l} \in \operatorname{gr}\left(X^{c}\right)$. Thus we have $\operatorname{gr}\left(X^{c}\right) \neq 1$ and the proof is complete. We remark the proof given does not cover the trivial case where $Y$ is a fan for all $Y \in \mathcal{U}(X)$. In this case, if $\sigma, \tau \in X^{c}$ are arbitrary then $\gamma=\sigma \tau \in \operatorname{gr}\left(X^{c}\right)$ so the result is valid in this case also.

THEOREM 2.6. Suppose $X$ is DLO finite and $n \geq 0$. Then the following are equivalent:

(i) $\sup \{|E(Y, \sigma)|: Y \in \mathcal{Q}(X), \sigma \in Y\} \leq n$. (Here $E(Y, \sigma)$ is defined as in [11].)

(ii) $X \in \mathscr{Q}_{n}$ where $\mathscr{Q}_{0}$ denotes the class of singleton spaces and, inductively, $2_{k+1}$ is the class of all DLO finite spaces $Y$ expressible as $Y \cong$ $\sum_{l \in I} Y_{l}^{\left(\alpha_{i}\right)}$ where $\alpha_{l}$ is a cardinal number and $Y_{l} \in \mathcal{Q}_{k}$ for all $i \in I$. (Here, $Y^{(\alpha)}$ denotes the group extension of $Y$ by the group $\{ \pm 1\}^{\alpha}$.)

Proof. This follows by induction using results in [11], but the same sort of proof as in Theorem 2.5.

EXAMPLE 2.7. The following illustrate why some finiteness conditions are necessary to get structure results like Theorems 2.5 and 2.6:

(i) $X=\left\{\sigma_{0}\right\} \oplus\left(\left\{\sigma_{1}\right\} \oplus\left(\left\{\sigma_{2}\right\} \oplus \cdots\right)^{(1)}\right)^{(1)}$. This satisfies $X \cong\left\{\sigma_{0}\right\} \oplus$ $X^{(1)}$, so if we attempt to decompose $X$ using Theorems 2.4 and 2.2 successively, we have "infinite descent". 
(ii) $X=\cdots\left(\left(\left\{\sigma_{0}\right\}^{(1)} \oplus\left\{\sigma_{1}\right\}\right)^{(1)} \oplus\left\{\sigma_{2}\right\}\right)^{(1)} \cdots$. This satisfies $\operatorname{gr}\left(X^{c}\right)=$ 1 but at the same time $X^{c}$ is connected, so both types of decomposition are "blocked" right from the start.

REMARK 2.8. It is not known if DLO finite spaces exist with $X^{c}$ connected, $|X| \neq 1$, and $\operatorname{gr}(X)=1$. The following shows that if $X$ is such a space then $X^{c}$ is uncountable. For suppose $X^{c}$ is countable. Then there exists a countable sequence

$$
X_{0} \subseteq X_{1} \subseteq \cdots
$$

where $X_{i}$ is a finite subspace of $X$ and $X^{c}=\cup_{i \geq 0} X_{i}$. Thus $X=\lim _{i \geq 0} X_{i}$. Since $X^{c}$ is connected we can choose $(*)$ so that $\operatorname{gr}\left(X_{i}\right) \neq 1$ for all $i \geq 0$. Denote by $\left[X_{i}\right]$ the linear span of $X_{i}$ in $\chi(G(X))$. First assume there exists $n \geq 1$ such that $\operatorname{gr}\left(X_{i}\right) \subseteq\left[X_{i-1}\right]$ for all $i \geq n$. Since $X_{i-1}$ is a subspace of $X_{i}$ this implies $\operatorname{gr}\left(X_{i}\right) \subseteq \operatorname{gr}\left(X_{i-1}\right)$ for $i \geq n$. Since these groups are finite and $\neq 1$, there exists $\gamma \in \bigcap_{i \geq n} \operatorname{gr}\left(X_{i}\right), \gamma \neq 1$. Thus $\gamma \in \operatorname{gr}\left(X^{c}\right) \subseteq \operatorname{gr}(X)$, so $\operatorname{gr}(X) \neq 1$. Now assume no such $n$ exists. Then, replacing $(*)$ by a suitable subsequence, we can assume $\operatorname{gr}\left(X_{i}\right) \not\left[X_{t-1}\right]$ for all $i \geq 1$. Thus there exists $a_{i} \in G(X)$ such that $a_{i} \in X_{i-1}^{\perp}$ but $a_{i} \notin \operatorname{gr}\left(X_{i}\right)^{\perp}$. Thus, if $b_{i-1} \in G(X)$ is arbitrary, there exists $b_{i} \in G(X)$ such that $b_{i} b_{i-1} \in X_{l-1}^{\perp}$ and $b_{i} \notin \operatorname{gr}\left(X_{i}\right)^{\perp}$. (Take $b_{i}=b_{i-1}$ if $b_{i-1} \notin \operatorname{gr}\left(X_{i}\right)^{\perp}$, and $b_{i}=a_{i} b_{t-1}$ if $b_{l-1} \in \operatorname{gr}\left(X_{i}\right)^{\perp}$.) Since $G(X)=\lim _{i \geq 0} G\left(X_{l}\right)$, this yields an element $x \in$ $G(X)$ satisfying $x_{i} \notin \operatorname{gr}\left(X_{i}\right)^{\perp}$ for all $i \geq 0$. Thus, by [10, Th. 6.6], and Corollary $1.2, x$ and $-x$ are both rigid and $\operatorname{gr}(X) \neq 1$.

3. Topological structure of $X^{c} \subseteq X$. For a space of orderings $X$ and $\sigma \in X$ the local stability index $\operatorname{st}(X, \sigma)$ is defined as in [11]. Thus, $\operatorname{st}(X)=\sup \{\operatorname{st}(X, \sigma): \sigma \in X\}$.

Theorem 3.1. Suppose $X$ is DLO finite and $\sigma \in X^{c}$. Then the following are equivalent:

(i) $\{\sigma\}$ is open in $X^{c}$,

(ii) $\{\sigma\}$ is open in $X$,

(iii) $\operatorname{st}(X, \sigma)<\infty$.

Further, $\operatorname{st}(X, \sigma)=\sup \{\operatorname{st}(Y, \sigma): Y \in \mathcal{Q}(X), \sigma \in Y\}$.

Proof. The equivalence of (i) and (ii) follows from the density of $X^{c}$ in $X$. Also, if $\{\sigma\}$ is open in $X$, then by definition of the topology on $X$, there exist $a_{1}, \ldots, a_{n} \in G(X)$ such that $X\left(a_{1}, \ldots, a_{n}\right)=\{\sigma\}$. (Recall $X\left(a_{1}, \ldots, a_{n}\right):=\left\{\tau \in X: \tau\left(a_{i}\right)=1\right.$ for all $\left.i=1, \ldots, n\right\}$.) Thus, by [11, Th. 3.4], (ii) $\Rightarrow$ (iii). Let $l=\sup \{\operatorname{st}(Y, \sigma): \quad Y \in \mathcal{Q}(X), \quad \sigma \in Y\}$. Clearly $\operatorname{st}(X, \sigma) \geq l$. Now suppose $l<\infty$. Suppose $\sigma \in Y$ where $Y \in \mathcal{Q}(X)$. 
Since $Y$ is finite, $\{\boldsymbol{\sigma}\}$ is open in $Y$ so by [11, Th. 3.4] there exist elements $a_{1 Y}, \ldots, a_{l Y} \in G(X)$ such that $Y\left(a_{1 Y}, \ldots, a_{l Y}\right)=\{\sigma\}$. Consider the Pfister form $p_{Y}=\left\langle 1, a_{1 Y}\right\rangle \otimes \cdots \otimes\left\langle 1, a_{I Y}\right\rangle$. By the proof of Theorem 1.3 there exists a form $p=\left\langle b_{1}, \ldots, b_{s}\right\rangle$ over $G(X)$ (where $s=2^{l}$ ) such that $p \cong_{Y} p_{Y}$ for all $Y \in \mathcal{Q}(X)$ such that $\sigma \in Y$. Note that $\sigma(p)=2^{l}$ whereas $\tau(p)=0$ for all $\tau \in X^{c}, \tau \neq \sigma$. It follows, in particular, that $2^{s-l} \times p$ and $\left\langle 1, b_{1}\right\rangle \otimes \cdots \otimes\left\langle 1, b_{s}\right\rangle$ have the same signature at all $\tau \in X^{c}$ and hence are isometric. Thus, applying [8, Lem. 6.3] repeatedly, $p$ is a Pfister form, say $p \cong\left\langle 1, a_{1}\right\rangle \otimes \cdots \otimes\left\langle 1, a_{l}\right\rangle$. Then it is clear that $X\left(a_{1}, \ldots, a_{l}\right) \cap X^{c}=$ $\{\sigma\}$ and hence that $X\left(a_{1}, \ldots, a_{l}\right)=\{\sigma\}$. This proves $\operatorname{st}(X, \sigma) \leq l$ and completes the proof.

Theorem 3.2. Suppose $X$ is DLO finite. Then the following are equivalent:

(i) $\operatorname{st}(X)<\infty$,

(ii) $X^{c}$ with the induced topology is discrete, and $X$ is the Stone-Čech compactification of $X^{c}$.

The proof of Theorem 3.2 uses another result which is of some independent interest:

THEOREM 3.3. Suppose $X$ is any space of orderings, $|X|>1$, and $f$ is a form over $G(X)$ satisfying the inequality $\operatorname{dim}(f)>|\sigma(f)| \cdot 2^{\mathrm{st}(X, \sigma)-1}$ for all $\sigma \in X$. (Here, we observe the convention $0 \cdot \infty=0$.) Then $f$ is isotropic.

Note, in particular, Theorem 3.3 asserts that, if $\operatorname{st}(X)=1$, then every totally indefinite form over $G(X)$ is isotropic. Theorem 3.3 should be viewed as a generalization of this well-known property of SAP spaces.

Proof of Theorem 3.3. By [9, Th. 1.4], we can assume $X$ is finite. The proof is by induction on the global stability index $\operatorname{st}(X)$. If $X=X_{1}$ $\oplus \cdots \oplus X_{s}$ and if the result is true for each $X_{1}$ with $\left|X_{l}\right|>1$, then it is true for $X$. Thus, by [9, Th. 1.3] we can assume $X=Y^{(1)}$, notation as in Theorem 2.5, and $|Y|>1$. Thus $f \cong f_{1} \oplus f_{2} t$ where $t$ is an element of $G(X) \backslash G(Y)$ and $f_{1}, f_{2}$ are forms over $G(Y)$. Scaling $f$ by $t$ if necessary, we can assume $\operatorname{dim}\left(f_{1}\right) \geq \operatorname{dim}\left(f_{2}\right)$. Let $\sigma \in Y$. Note if $e \in\{ \pm 1\}$ is suitably chosen, then $\left|\sigma\left(f_{1}\right)+e \sigma\left(f_{2}\right)\right| \geq\left|\sigma\left(f_{1}\right)\right|$. Extend $\sigma$ to an element $\tau \in X$ in such a way that $\tau(t)=e$. Thus $|\tau(f)| \geq\left|\sigma\left(f_{1}\right)\right|$. Also $\operatorname{st}(X, \tau)=\operatorname{st}(Y, \sigma)$ +1 so

$$
2 \cdot \operatorname{dim}\left(f_{1}\right) \geq \operatorname{dim}(f)>|\tau(f)| \cdot 2^{\mathrm{st}(X, \tau)-1} \geq\left|\boldsymbol{\sigma}\left(f_{1}\right)\right| \cdot 2^{\mathrm{st}(Y, \sigma)}
$$


so

$$
\operatorname{dim}\left(f_{1}\right)>\left|\sigma\left(f_{1}\right)\right| \cdot 2^{\mathrm{st}(Y, \sigma)-1}
$$

for all $\sigma \in Y$. Finally, st $(Y)=\operatorname{st}(X)-1$ so by induction on the stability index, $f_{1}$ is $Y$-isotropic, so $f$ is $X$-isotropic.

Proof of Theorem 3.2. Assume (i), say $\operatorname{st}(X)=2^{k}<\infty$. Thus, by Theorem 3.1, $X^{c}$ is discrete in $X$. To show $X$ is the Stone-Čech compactification of $X^{c}$, we show that every function $\varphi: X^{c} \rightarrow\{ \pm 1\}$ extends to a continuous function $\psi: X \rightarrow\{ \pm 1\}$. Consider $2^{k} \varphi: X^{c} \rightarrow \mathbf{Z}$. By [8, Ths. 5.5 and 6.4], for all $Y \in \mathcal{Q}(X)$, there exists an anisotropic form $f_{Y}$ over $G(Y)$ representing $2^{k} \varphi$. Thus, by Theorem 3.3, there exists $\sigma \in Y$ such that

$$
\operatorname{dim}\left(f_{Y}\right) \leq\left|\sigma\left(f_{Y}\right)\right| \cdot 2^{\mathrm{st}(Y)-1} \leq 2^{k} 2^{k-1}=2^{2 k-1},
$$

so by Theorem 1.3, there exists a form $f$ over $G(X)$ such that $\sigma(f)=$ $2^{k} \varphi(\sigma)$ for all $\sigma \in X^{c}$. Define $\psi: X \rightarrow\{ \pm 1\}$ by $\psi(\sigma)=2^{-k} \sigma(f)$ for all $\sigma \in X$. This is continuous and extends $\varphi$.

Suppose (ii) holds but (i) fails. Thus

$$
\begin{aligned}
\operatorname{st}(X) & =\sup \{\operatorname{st}(Y): Y \in \mathcal{U}(X)\} \\
& =\sup \{\operatorname{st}(Y, \sigma): Y \in \mathcal{U}(X), \sigma \in Y\}=\infty
\end{aligned}
$$

so there are arbitrarily large finite fans in $X^{c}$, but, by Theorem 3.1, $\operatorname{st}(X, \sigma)<\infty$ holds for all $\sigma \in X^{c}$. These facts can be used to construct a sequence of fans $V_{1}, V_{2}, \ldots$ in $X^{c}$ of increasing size and mutually disjoint. (For if $V_{1}, \ldots, V_{k-1}$ are already constructed just let $m=\sup \{\operatorname{st}(X, \sigma)$ : $\left.\sigma \in V_{1} \cup \cdots \cup V_{k-1}\right\}$ and pick $V_{k}$ to be any fan in $X^{c}$ with $\left|V_{k}\right|>2^{m}$. then $V_{k} \cap V_{i}=\varnothing$ for $i=1, \ldots, k-1$.) Now pick $\sigma_{i} \in V_{i}$ for each $i \geq 1$, and define $\varphi: X^{c} \rightarrow\{0,1\}$ by $\varphi\left(\sigma_{l}\right)=1$ for $i=1,2, \ldots$ and $\varphi(\sigma)=0$ if $\sigma \notin\left\{\sigma_{1}, \sigma_{2}, \ldots\right\}$. This extends to a continuous $\psi: X \rightarrow\{0,1\}$ so by [8, Lem. 5.4] there exists $k \geq 0$ and a form $f$ over $G(X)$ such that $\sigma(f)=2^{k}$. $\psi(\sigma)$ for all $\sigma \in X$. This contradicts [8, Th. 5.5] since if $i$ is sufficiently large, $\left|V_{i}\right|>2^{k}$ so $\Sigma_{\sigma \in V_{i}} \sigma(f)=2^{k} \neq 0 \bmod \left|V_{i}\right|$.

Corollary 3.4. Suppose $X$ is DLO finite, $\operatorname{st}(X)<\infty$, and $\varphi: X^{c} \rightarrow \mathbf{Z}$ is any function. Then the following are equivalent:

(i) There exists a form $f$ over $G(X)$ such that $\sigma(f)=\varphi(\sigma)$ for all $\sigma \in X^{c}$.

(ii) $\varphi\left(X^{c}\right)$ is a finite set and $\Sigma_{\sigma \in V} \varphi(\sigma) \equiv 0 \bmod |V|$ holds for all finite fans $V \subseteq X^{c}$. 
Proof. (i) $\Rightarrow$ (ii) is true by [8, Th. 5.5]. (ii) $\Rightarrow$ (i) follows by an argument similar to the first half of the proof of Theorem 3.2.

EXAMPLE 3.5. We conclude this section with an example of how Corollary 3.4 can fail if $\operatorname{st}(X)=\infty$. Define $X_{1}=\{\sigma\}$ and for each $i \geq 1$ define $X_{i+1}=\left(X_{i} \oplus X_{i}\right)^{(1)}$, notation as in Theorem 2.5. Thus $X=\sum_{t=1}^{\infty} X_{t}$ is DLO finite by Theorem 2.3. We claim there exists an anisotropic form $f_{l}$ over $G\left(X_{l}\right)$ such that $\operatorname{dim}\left(f_{i}\right)=2^{i}$ and $\sigma\left(f_{i}\right)= \pm 2$ for all $\sigma \in X_{l}$. Define $f_{1}=\langle 1,1\rangle$. Suppose $f_{l}$ is defined. Let $h_{i}$ be the hyperbolic form over $G\left(X_{t}\right)$ with dimension equal to $2^{i}=\operatorname{dim}\left(f_{l}\right)$. Define forms $f_{l}^{\prime}, f_{l}^{\prime \prime}$ over $G\left(X_{t} \oplus X_{i}\right)$ by $f_{l}^{\prime}=\left(f_{i}, h_{l}\right)$ and $f_{i}^{\prime \prime}=\left(h_{l}, f_{i}\right)$. Finally, define $f_{i+1}=f_{l}^{\prime} \oplus$ $f_{l}^{\prime \prime} t_{l}$ where $t_{i}$ is an element of $G\left(X_{l+1}\right) \backslash G\left(X_{i} \oplus X_{i}\right)$. This proves the claim. Since $X^{c}=\cup_{l=1}^{\infty} X_{l}$ by Theorem 2.3, the function $\varphi: X^{c} \rightarrow \mathbf{Z}$, defined by $\varphi\left(\sigma_{i}\right)=\sigma_{i}\left(f_{t}\right)$ if $\sigma_{t} \in X_{l}$, is well defined. Since $\varphi(\sigma)= \pm 2$ and since each non-trivial fan in $X^{c}$ lies in some $X_{l}, \varphi$ satisfies condition (ii) of Corollary 3.4. However, since $f_{l}$ is anisotropic of dimension $2^{t}$, there cannot be a form $f$ over $G(X)$ satisfying (i) of Corollary 3.4. Note, by Theorem 3.1, $X^{c}$ is discrete in $X$, so $\varphi$ is even continuous.

4. Direct limits and ultrasums. Recall that an ultrafilter $\mathscr{U}$ on a set $I$ is a collection of subsets of $I$ satisfying: (i) $\varnothing \notin \mathscr{Q}$ (ii) if $J, K \in \mathscr{Q}$ then $J \cap K \in \mathcal{Q}$, and (iii) if $J \notin \mathcal{Q}$, then $I \backslash J \in \mathcal{Q}$.

Let $\left\{X_{i}: i \in I\right\}$ be any collection of spaces of orderings, let $X=$ $\sum_{l \in I} X_{l}$, and if $J \subseteq I$, let $X_{J}=\sum_{i \in J} X_{i}$. Thus, if $K=I \backslash J$, then $X=X_{J} \oplus$ $X_{K}$ so, in particular, $X_{J}$ is a subspace of $X$. Thus, if $\mathscr{Q}$ is any ultrafilter on $I$, then $X_{\mathcal{Q}}:=\bigcap_{J \in \mathcal{Q}} X_{J}$ is a subspace of $X$. We refer to $X_{\mathcal{Q}}$ as the ultrasum of the collection $\left\{X_{i}: i \in I\right\}$ determined by $\mathscr{Q}$. Note that $G\left(X_{\mathscr{Q}}\right)=G(X) / H_{थ}$, where $H_{\mathscr{Q}}$ is the subgroup consisting of all $x \in$ $G(X)$ satisfying $\left\{i \in I: x_{i}=1\right\} \in \mathcal{Q}$, and $W\left(X_{\mathscr{Q}}\right)=W(X) / J_{\mathscr{Q}}$, where $J_{\mathscr{Q}}$ is the ideal consisting of all $f \in W(X)$ satisfying $\left\{i \in I: f_{i} \sim 0\right\} \in \mathcal{Q}$.

REMARK 4.1. Suppose we denote by $\beta(I)$ the Stone-Čech compactification of the discrete set $I$. Thus the clopen subsets of $\beta(I)$ are in 1-1 correspondence with the subsets of $I$ and the points of $\beta(I)$ are in 1-1 correspondence with the ultrafilters on $I$. One can verify that the Witt rings $W\left(X_{J}\right), J \subseteq I$, form a sheaf of Witt rings over $\beta(I)$ in the terminology of $[10, C h .8]$. The stalks of this sheaf are the Witt rings $W\left(X_{\mathscr{Q}}\right)$, Q $\in \beta(I)$.

LEMma 4.2. Suppose $K$ is the ultraproduct of the Pythagorean fields $\left\{K_{i}\right.$ : $i \in I\}$ determined by some ultrafilter $\mathcal{Q}$. Then $K$ is a Pythagorean field and 
$X_{K}$ is naturally identified with the ultrasum $\left(\Sigma_{i \in I} X_{K_{1}}\right)_{\text {थ. }}$. (Here, $X_{K}$ denotes the space of orderings of the field $K$ ).

Proof. By definition, $K$ is the ring $\left(\prod_{i \in I} K_{l}\right) / M_{\mathscr{Q}}$ where $M_{\mathcal{Q}}$ is the ideal consisting of all $a \in \Pi_{l \in I} K_{l}$ such that $\left\{i \in I: a_{l}=0\right\} \in \mathcal{Q}$. The check that $K$ is a Pythagorean field is straightforward. For the second assertion, one simply verifies that the natural map from $\dot{K} / \dot{K}^{2}$ into $\left(\prod_{l \in I} \dot{K}_{i} / \dot{K}_{i}^{2}\right) / H_{\mathscr{U}}$ is a group isomorphism which preserves value sets of binary forms. The result then follows from [8, Lem. 4.1]. More explicitly, it follows from

Lemma 4.3. Suppose $X, Y$ are spaces of orderings. Then a group homomorphism $\alpha: G(Y) \rightarrow G(X)$ induces a morphism $\alpha^{*}: X \rightarrow Y$ iff $\alpha(-1)$ $=-1$ and $\alpha\left(D_{Y}\langle 1, a\rangle\right) \subseteq D_{X}\langle 1, \alpha(a)\rangle$ for all $a \in G(Y)$.

Proof. One implication is clear. The other follows from [8, Lem. 4.1].

In case $I$ is a directed set, ultrafilters compatible with the ordering on $I$ are of special interest. These are just ultrafilters $\mathscr{U}$ on $I$ with the property that for all $i \in I$, the set $I(i):=\{j \in I: j \geq i\}$ belongs to $\mathcal{Q}$. Such ultrafilters exist by an easy application of Zorn's Lemma.

LEMMA 4.4. Suppose $\left(X_{i}: i \in I\right)$ is a directed system of finite spaces of orderings and $\mathcal{Q}$ is an ultrafilter on I compatible with the ordering. Then there are natural morphisms $\alpha^{*}:\left(\sum_{i \in I} X_{i}\right)_{\mathcal{Q}} \rightarrow \lim _{\rightarrow \in I} X_{i}$ and $\beta^{*}: \lim _{i \in I} X_{l}$ $\rightarrow\left(\sum_{t \in I} X_{t}\right)_{\mathscr{Q}}$ such that $\alpha^{*} \circ \beta^{*}=$ the identity.

Proof. Let $X=\Sigma_{l \in I} X_{i} . \alpha^{*}$ is just the composite of the inclusion $X_{थ} \subseteq X$ followed by the projection $X \rightarrow \lim _{i \in I} X_{i}$. The definition of $\beta^{*}$ is a bit more interesting. First note that, by the universal property of the direct limit, it is enough to define morphisms $\beta_{i}^{*}: X_{l} \rightarrow X_{\mathscr{Q}}$ such that, if $i \leq j$, then $\beta_{i}^{*}=\beta_{j}^{*} \circ \varphi_{i j}^{*}$ where the $\varphi_{i j}^{*}: X_{i} \rightarrow X_{j}$ are the morphisms defining the directed system. Let $x \in G(X)$. For fixed $y \in G\left(X_{i}\right)$ define $I(i, y)=\left\{j \in I: j \geq i\right.$ and $\left.\varphi_{i j}\left(x_{j}\right)=y\right\}$. Since $G\left(X_{i}\right)$ is finite, $I(i) \in \mathcal{Q}$, and $I(i)$ is the disjoint union of the sets $I(i, y), y \in G\left(X_{i}\right)$, it follows that there exists a unique $\beta_{i}(x) \in G\left(X_{i}\right)$ such that $I\left(i, \beta_{i}(x)\right) \in \mathcal{Q}$. Observe that $\beta_{i}: G(X) \rightarrow G\left(X_{l}\right)$ is a group homomorphism, $\beta_{i}(-1)=-1$, and if $y \in D\langle 1, x\rangle$, then $\beta_{i}(y) \in D\left\langle 1, \beta_{i}(x)\right\rangle$. Thus $\beta_{i}$ induces a morphism $\beta_{i}^{*}$ : $X_{l} \rightarrow X$ by Lemma 4.3. Note that the kernel of $\beta_{l}$ contains all $x$ such that $\left\{j \in I: x_{J}=1\right\} \in \mathcal{Q}$ so $\beta_{i}^{*}: X_{\imath} \rightarrow X_{\mathscr{Q}}$. Finally, if $j \geq i$, then $\beta_{i}=\varphi_{i j} \circ \beta_{j}$ 
so $\beta_{i}^{*}=\beta_{J}^{*} \circ \varphi_{i j}^{*}$. This completes the definition of $\beta^{*}$. Observe that if $x \in \lim _{i \in I} G\left(X_{i}\right)$, then $\beta_{j}(x)=x_{j}$. Thus $\alpha^{*} \circ \beta^{*}=1$.

TheOREM 4.5. Suppose $X$ is any DLO finite space. Then there exists a Pythagorean field $K$ and morphisms $\alpha^{*}: X_{K} \rightarrow X, \beta^{*}: X \rightarrow X_{K}$ such that $\alpha^{*} \circ \beta^{*}=1$.

Proof. Suppose $X=\lim _{\rightarrow \in I} X_{i}$ where each $X_{i}$ is finite. By [1] or [4] there exists a Pythagorean field $K_{i}$ with $X_{K_{l}} \cong X_{l}$. The result follows by applying Lemmas 4.2 and 4.4 .

To analyze further the conclusion of Theorem 4.5 we note the following:

LEMMA 4.6 Suppose $X, Y$ are spaces of orderings and $\alpha^{*}: X \rightarrow Y$ and $\beta^{*}: Y \rightarrow X$ are morphisms satisfying $\alpha^{*} \circ \beta^{*}=1$. Then

(i) $\alpha^{*}$ identifies $Y$ with a quotient space of $X$, and for any form $f$ over $G(Y)$, if $\alpha(f)$ is isotropic then $f$ is isotropic, and

(ii) $\beta^{*}$ identifies $Y$ with a subspace of $X$.

Proof. The hypothesis implies $\alpha^{*}$ is onto and $\beta^{*}$ is 1-1. It also implies $\beta \circ \alpha=1$ so $\alpha$ is $1-1$ and $\beta$ is onto. The assertion that $Y$ is a quotient space of $X$ is now clear. Also, if $\alpha(f)$ is isotropic, then so is $\beta(\alpha(f))=f$, so (i) is clear. To complete the proof of (ii), it is necessary to check that $\beta^{*}(Y)$ is a subspace of $X$. This amounts to showing that if $\sigma \in X$ satisfies $\sigma(a)=1$ for all $a \in \operatorname{ker}(\beta)$, then $\sigma \in \beta^{*}(Y)$. Let $b \in G(X)$. Then $\beta(\alpha(\beta(b)))=\beta(b)$ so $\alpha(\beta(b)) \cdot b \in \operatorname{ker}(\beta)$. Thus $\sigma(\alpha(\beta(b)) \cdot b)=1$, i.e.,

$$
\sigma(b)=\sigma(\alpha(\beta(b)))=\left(\beta^{*}\left(\alpha^{*}(\sigma)\right)\right)(b) .
$$

Since $b \in G(X)$ is arbitrary, this implies $\sigma=\beta^{*}\left(\alpha^{*}(\sigma)\right)$, so $\sigma \in \beta^{*}(Y)$.

COROLlaRY 4.7. Every DLO finite space of orderings is isomorphic to a subspace of the space of orderings of a suitably chosen Pythagorean field.

Proof. Immediate from Theorem 4.5 and Lemma 4.6(ii).

COROLlary 4.8. Every space of orderings $X$ is isomorphic to a quotient space of the space of orderings of a suitably chosen Pythagorean field $K$. Further, this representation of $X$ can be achieved in such a way that for a form $f$ over $G(X), f$ is $X$-isotropic iff $f$ is $X_{K}$-isotropic.

Proof. Immediate from Remark 1.4, Theorem 4.5, and Lemma 4.6(i). 


\section{REFERENCES}

[1] T. Graven, Characterizing reduced Witt rings of fields, J. Algebra, 53 (1978), 68-77.

[2] __ Characterizing reduced Witt rings II, Pacific J. Math., 80 (1979), 341-349.

[3] J. Kleinstein and A. Rosenberg, Succinct and representational Witt rings, Pacific J. Math., 86 (1980), 99-137.

[4] M. Kula, Fields with prescribed quadratic form schemes, Math. Zeit., 167 (1979), 201-212.

[5] _ Finite sets of quasi-orderings, Prace Mat. 10, Prace nankowe Uniwersytetu Slaskiego, 332 (1980), 69-85.

[6] M. Marshall, Classification of finite spaces of orderings, Canad. J. Math., 21(2) (1979), $320-330$.

[7] _ Quotients and inverse limits of spaces of orderings, Canad. J. Math., 31(3) (1979), 604-616.

[8] The Witt ring of a space of orderings, Trans. Amer. Math. Soc., 258 (1980), 505-521.

[9] _ Spaces of orderings IV, Canad. J. Math., 32(3) (1980), 603-627.

[10] Abstract Witt rings, Queen's Papers in Pure and App. Math. \#57, Queen's University, Kingston, Canada.

[11] Spaces of orderings: systems of quadratic forms, local structure, and saturation, Comm. in Algebra, to appear.

[12] A. Sładek, Abstract theory of quadratic forms, Prace Mat. 10, Prace nankowe Uniwersytetu Slaskiego, 332 (1980), 46-57.

Received October 14, 1981 and in revised form May 9, 1983.

Mieczyslaw Kula and Andrzej Sladek

SILESIAN UNIVERSITY

Katowice, Poland

AND

Murray Marshall

UNIVERSITY OF SASKATCHEWAN

Saskatoon, Canada 


\title{
PACIFIC JOURNAL OF MATHEMATICS EDITORS
}

\author{
Donald BabBITT (Managing Editor) \\ University of California \\ Los Angeles, CA 90024 \\ Hugo Rossi \\ University of Utah \\ Salt Lake City, UT 84112 \\ C. C. Moore and Arthur Ogus \\ University of California \\ Berkeley, CA 94720
}

\author{
J. Dugundu \\ Department of Mathematics \\ University of Southern California \\ Los Angeles, CA 90089-1113
}

R. FINN and H. SAMELSON

Stanford University

Stanford, CA 94305

\section{ASSOCIATE EDITORS}
R. ARENS
E. F. BECKENBACH
B. H. NeumanN
F. WolF
K. YosHIDA (1906-1982)

\section{SUPPORTING INSTITUTIONS}

\author{
UNIVERSITY OF ARIZONA \\ UNIVERSITY OF BRITISH COLUMBIA \\ CALIFORNIA INSTITUTE OF TECHNOLOGY \\ UNIVERSITY OF CALIFORNIA \\ MONTANA STATE UNIVERSITY \\ UNIVERSITY OF NEVADA, RENO \\ NEW MEXICO STATE UNIVERSITY \\ OREGON STATE UNIVERSITY
}

\author{
UNIVERSITY OF OREGON \\ UNIVERSITY OF SOUTHERN CALIFORNIA \\ STANFORD UNIVERSITY \\ UNIVERSITY OF HAWAII \\ UNIVERSITY OF TOKYO \\ UNIVERSITY OF UTAH \\ WASHINGTON STATE UNIVERSITY \\ UNIVERSITY OF WASHINGTON
}

The Supporting Institutions listed above contribute to the cost of publication of this Journal, but they are not owners or publishers and have no responsibility for its content or policies.

Mathematical papers intended for publication in the Pacific Journal of Mathematics should be in typed form or offset-reproduced (not dittoed), double spaced with large margins. Please do not use built up fractions in the text of the manuscript. However, you may use them in the displayed equations. Underline Greek letters in red, German in green, and script in blue. The first paragraph must be capable of being used separately as a synopsis of the entire paper. In particular it should contain no bibliographic references. Please propose a heading for the odd numbered pages of less than 35 characters. Manuscripts, in triplicate, may be sent to any one of the editors. Please classify according to the scheme of Math. Reviews, Index to Vol. 39. Supply name and address of author to whom proofs should be sent. All other communications should be addressed to the managing editor, or Elaine Barth, University of California, Los Angeles, California 90024.

There are page-charges associated with articles appearing in the Pacific Journal of Mathematics. These charges are expected to be paid by the author's University, Government Agency or Company. If the author or authors do not have access to such Institutional support these charges are waived. Single authors will receive 50 free reprints; joint authors will receive a total of 100 free reprints. Additional copies may be obtained at cost in multiples of 50 .

The Pacific Journal of Mathematics is issued monthly as of January 1966. Regular subscription rate: $\$ 132.00$ a year (6 Vol., 12 issues). Special rate: $\$ 66.00$ a year to individual members of supporting institutions.

Subscriptions, orders for numbers issued in the last three calendar years, and changes of address should be sent to Pacific Journal of Mathematics, P.O. Box 969, Carmel Valley, CA 93924, U.S.A. Old back numbers obtainable from Kraus Periodicals Co., Route 100, Millwood, NY 10546.

The Pacific Journal of Mathematics ISSN 0030-8730 is published monthly by the Pacific Journal of Mathematics at P.O. Box 969, Carmel Valley, CA 93924. Application to mail at Second-class postage rates is pending at Carmel Valley, California, and additional mailing offices. Postmaster: Send address changes to Pacific Journal of Mathematics, P. O. Box 969, Carmel Valley, CA 93924.

PUBLISHED BY PACIFIC JOURNAL OF MATHEMATICS. A NON-PROFIT CORPORATION

Copyright $\odot 1984$ by Pacific Journal of Mathematics 


\section{Pacific Journal of Mathematics}

\section{Vol. 112, No. $2 \quad$ February, 1984}

Kenneth F. Andersen and Wo-Sang Young, On the reverse weak type inequality for the Hardy maximal function and the weighted classes

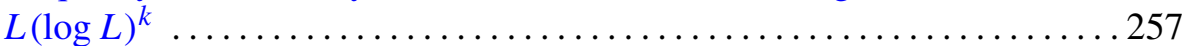

Richard Eugene Bedient, Double branched covers and pretzel knots ..... 265

Harold Philip Boas, Holomorphic reproducing kernels in Reinhardt

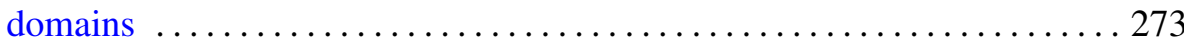

Janey Antonio Daccach and Arthur Gabriel Wasserman, Stiefel's theorem and toral actions ................................. 293

Michael Fried, The nonregular analogue of Tchebotarev's theorem ....... 303

Stanley Joseph Gurak, Minimal polynomials for circular numbers . . . . . . 313

Norimichi Hirano and Wataru Takahashi, Nonlinear ergodic theorems for an amenable semigroup of nonexpansive mappings in a Banach space . . 333

Jim Hoste, Sewn-up $r$-link exteriors . . . . . . . . . . . . . . . . . . . 347

Mohammad Ahmad Khan, The existence of totally dense subgroups in

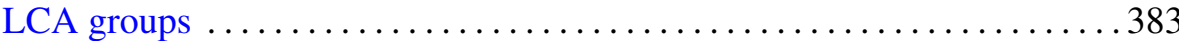

Mieczysław Kula, Murray Angus Marshall and Andrzej Sładek, Direct

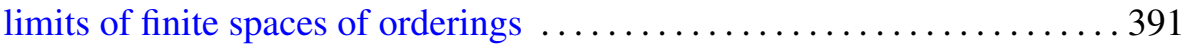

Luis Montejano Peimbert, Flat Hilbert cube manifold pairs ........... 407

Steven C. Pinault, An a priori estimate in the calculus of variations . . . . . 427

McKenzie Y. K. Wang, Some remarks on the calculation of Stiefel-Whitney classes and a paper of Wu-Yi Hsiang's

Brian Donald Wick, The calculation of an invariant for Tor . . 445

Wolfgang Wollny, Contributions to Hilbert's eighteenth problem 